\title{
From the Desk of the Editors
}

\section{- Student Research}

The following papers are presented to demonstrate our desire to encourage the submission of research activities completed by students in all types of educational programs. We have tried to use papers that are representative of student efforts in the realm of exercise science. It is our hope to include a group of student papers on a somewhat regular basis.

Please foster the submission of papers by your students. IES

George J. Davies, Terry R. Malone, and Kent Timm 